\title{
Differential Yellow Fever Susceptibility in New World Nonhuman Primates, Comparison with Humans, and Implications for Surveillance
}

\author{
Natália C.C. de Azevedo Fernandes, Juliana M. Guerra, Josué Díaz-Delgado, Mariana S. Cunha, \\ Leila del C. Saad, Silvia D. Iglezias, Rodrigo A. Ressio, Cinthya dos Santos Cirqueira, Cristina T. Kanamura, \\ Isis P. Jesus, Adriana Y. Maeda, Fernanda G.S. Vasami, Júlia de Carvalho, Leonardo J.T. de Araújo, \\ Renato Pereira de Souza, Juliana S. Nogueira, Roberta M.F. Spinola, José L. Catão-Dias
}

A major outbreak of yellow fever (YF) occurred in Brazil during 2016-2018. Epizootics in New World nonhuman primates are sentinel events for YF virus circulation. However, genus-specific susceptibilities and suitability for YF surveillance remain poorly understood. We obtained and compared epidemiologic, histopathologic, immunohistochemical, and molecular results from 93 human and 1,752 primate cases submitted during the recent YF outbreak in Brazil (2017), with the support of the Brazilian National YF Surveillance Program. We detected heterogeneous YF-associated profiles among the various genera of primates we analyzed. Alouatta primates were the most reliable sentinel; Sapajus and Callicebus primates had higher viral loads but lower proportional mortality rates. Callithrix primates were the least sensitive, showing lower viral loads, lower proportional mortality rates, and no demonstrable YF virus antigen or extensive lesions in liver, despite detectable viral RNA. These differences in susceptibility, viral load, and mortality rates should be considered in strategic surveillance of epizootics and control measures for YF.

Author affilations: Instituto Adolfo Lutz, São Paulo, Brazil (N.C.C. de Azevedo Fernandes, J.M. Guerra, J. Díaz-Delgado, M.S. Cunha, S.D. Iglezias, R.A. Ressio, C. dos Santos Cirqueira, C.T. Kanamura, I.P. Jesus, A.Y. Maeda, F.G.S. Vasami,

J. de Carvalho, L.T. de Araújo, R. Pereira de Souza,

J.S. Nogueira); Universidade de São Paulo, São Paulo

(N.C.C. de Azevedo Fernandes, J.M. Guerra, J. Díaz-Delgado,

J.L. Catão-Dias); Texas A\&M Veterinary Medical Diagnostic

Laboratory, College Station, Texas, USA (J. Díaz-Delgado); Centro

de Vigilância Epidemiológica Prof. Alexandre Vranjac, São Paulo

(L.D.C. Saad, R.M.F. Spinola)

DOI: https://doi.org/10.3201/eid2701.191220
Vellow fever (YF) is a zoonosis caused by YF virus (YFV; family Flaviviridae, genus Flavivirus) that has 2 established cycles in South America: urban and sylvatic. The sylvatic cycle is maintained by forest canopy mosquitoes (Sabethes spp. and Haemagogus spp.) and New World primates (NWPs); humans are accidental hosts (1,2). During 2016-2018, YF reemerged in Brazil, posing new threats with major epidemic waves in areas with low viral circulation, although without evidence of the urban cycle (3). The national surveillance program in Brazil for YF primarily relies on YF investigation in deceased free-ranging NWPs (4). Surveillance of epizootics in NWPs plays a pivotal role for deployment of prevention actions, emphasizing immediate vaccination of susceptible human populations (5).

Brazil has broad and heterogeneous NWP (suborder Platyrrhini) diversity: 5 families, 21 genera, and 176 species (6). Nevertheless, knowledge of YF in NWP species is limited; most efforts have focused on serologic testing of the species Leontopithecus chrysomelas (7), Alouatta spp. (8,9), Cebus spp.(10), and Saguinus spp. and Saimiri spp. (10). Only howler monkeys (Alouatta sp.) have well-documented liver involvement in consequence of natural YF infection (11) and are considered reliable sentinels of YFV circulation, because they show higher susceptibility to YF than humans and develop a fatal hepatic failure with massive cellular death $(1,4,12)$. Howler monkeys and laboratory NWP models usually have hepatic changes similar to those found in humans $(3,11)$ : massive necrosis/apoptosis associated with CouncilmanRocha Lima bodies, steatosis, and mild inflammatory 
infiltrates $(13,14)$, although diverging pathologic features might also be seen (3). Further knowledge of YF pathogenic aspects is needed to guarantee that samples from various species of NWPs would be adequately used for YF surveillance purposes to ensure appropriate diagnoses and subsequent public health responses. In addition, this knowledge will clarify the effect of YF in the wide range of NWPs in Brazil.

During the last YF outbreak in Brazil (2016-2018), we observed differences among genera not only in YF prevalence but also in YF viral load, as reported (15). These differences might have implications for NWP as amplifying hosts or as reservoirs in YF cycle. The term reservoir refers to an animal with persistent infection, sometimes without clinical signs, and sufficient amount of pathogen to act as source of infection. Amplifier, although used as a synonym, is a broad term referring to a host with high viral load and source of infection (16). Thus, we hypothesized that there are different genus-specific susceptibilities among NWPs that could affect YF surveillance/monitoring. To reduce this knowledge gap, we characterized and compared the histopathologic signature of YF-associated liver disease, viral antigen detection by immunohistochemical analysis (IHC), and molecular findings in samples from humans and NWPs infected by YFV that were received during 2017 at Adolfo Lutz Institute (São Paulo, Brazil).

\section{Methods}

\section{Data and Sample Collection}

We obtained formalin-fixed, paraffin-embedded and fresh frozen $\left(-70^{\circ} \mathrm{C}\right)$ liver samples from NWPs and humans from São Paulo state, Brazil, that were submitted for YF diagnosis to the Adolfo Lutz Institute during 2017, according to the Brazilian National Surveillance Program of YF by the Ministry of Health of Brazil (4). The Adolfo Lutz Institute is an official laboratory for the diagnosis of YFV in humans and primates. NWP samples came from standardized necropsies performed by local surveillance agents. Epidemiologic (carcass location and date) and biological (genera, sex, age) data were obtained from notification files (Sistema Nacional de Agravos de Notificação) sent with the samples. Cases designated as Cebus spp. were reclassified as Sapajus spp., according to Alfaro et al. (17). Only NWP cases with genera identified and formalin-fixed, paraffin-embedded liver tissue were included in this study. For humans, samples from patients with suspected or confirmed YF who died were obtained; only cases with quantitative reverse transcription PCR (qRT-PCR) results from fresh liver samples were included. Adverse vaccine effects and transplant cases were excluded. All procedures were approved by the Animal Use and Research Ethical Committees of the Adolfo Lutz Institute (CEUAIAL no. 11/2016 and CEP-IAL no. 3.121.328-caaee 96138818.0.0000.0059) and the Instituto Chico Mendes de Conservação da Biodiversidade protocol 50551-3.

\section{Spatial Analysis}

Cases positive by IHC or qRT-PCR were tabulated and plotted. Plots were made by using a map of São Paulo state and QGis software (https:/ / qgis.org).

\section{Histopathologic and Immunohistochemical Analyses}

Protocols and procedures were conducted in the enhanced laboratory Biosafety Level 2 facility of the Adolfo Lutz Institute. All formalin-fixed, paraffin-embedded liver tissue samples were processed and stained with hematoxylin and eosin for histopathologic examination. IHC was performed according to our laboratory protocols. Liver tissue sections were subjected to antigen retrieval in a pressure cooker in citrate buffer for $3 \mathrm{~min}\left(120^{\circ} \mathrm{C}, \mathrm{pH} 6.0\right)$ and then incubated overnight with polyclonal anti-YF (mouse hyperimmune antiserum against wild strain; Núcleo de Doença de Transmissão Vetorial, Virology Center, Adolfo Lutz Institute) $(3,12)$. Signal amplification was performed by using the Horseradish Peroxidase-Conjugated Polymer Detection System (REVEAL Biotin-Free Polyvalent; Spring Bioscience Corp., https://www. cmocro.com) and visualized by using diaminobenzidine (D-5637; Sigma-Aldrich, https://www.sigmaaldrich.com) and counterstaining with Harris hematoxylin. In selected instances, amplification was performed by using AP conjugated polymer (MACH4 Universal AP Polymer Kit; Biocare Medical, https://biocare.net) and visualized by using fast red chromogen (WARP RED chromogen kit; Biocare Medical). Known NWPs and human positive and negative control tissues with omitted first-layer antibody were included.

NWP cases were classified according to the distribution, extent, and nature of microscopic findings after staining with hematoxylin and eosin as described (Appendix Table 1, https://wwwnc.cdc.gov/EID/ article/27/1/19-1220-App1.pdf). For IHC, cases were classified as positive, negative, or inadequate on the basis of YFV antigen detection and varying degrees of typical YF-associated lesions. Inadequate classification refers to highly autolyzed/decomposed cases with lack of immunolabeling. Human cases were classified as full spectrum of YF-associated hepatic lesions or other histologic patterns. We provide a detailed description of other histologic patterns. 


\section{Molecular Analysis by Using qRT-PCR}

Total RNA was extracted from fresh frozen liver by using the QIAamp RNA Blood Mini Kit (QIAGEN, https://www.qiagen.com), according to the manufacturer's instructions. Amplification of YFV fragments were performed by using a described protocol (18) that targets the highly conserved 5' noncoding region of the genome (112 bp) and is based on a TaqMan qRT-PCR protocol. Quantification cycle (Cq) was used as a reference for viral load (in a standard curve with YFV vaccine 17D, a titer of $10^{5} \mathrm{PFU} / \mathrm{mL}$ had a mean $\mathrm{Cq}=16$, and $1 \mathrm{PFU} / \mathrm{mL}$ had a mean $\mathrm{Cq}=35.6$ ).

\section{Statistical Analysis}

Data were tabulated and analyzed by using Portal Action (http://www.portalaction.com.br) and R software (https://www.r-project.org). For categorical data, we used $\chi^{2}$ or Fisher exact test (2-tailed) tests. For continuous data, after using the ShapiroWilk normality test, we applied the Kruskal-Wallis test. Agreement between IHC and qRT-PCR was calculated by using the Cohen kappa contingency coefficient. Sensitivity, specificity, positive predictive value, negative predictive value, and accuracy of histologic analysis were calculated; inadequate cases obtained by IHC were excluded. The IHC detection limit (qRT-PCR Cq value) was obtained by using receiver operating characteristic curve analysis. Correlation of nonparametric data was calculated by using the Spearman test, and a standard curve was created. Statistical difference was defined as $\mathrm{p}<0.05$.

\section{Results}

\section{NWPs}

We analyzed 1,752 NWPs: 413 (23.6\%) were female, $638(36.4 \%)$ were male, and $701(40 \%)$ did not have sex identified. Distribution by genus was 921 (52.6\%) Callithrix spp., 708 (40.4\%) Alouatta spp., 95 (5.4\%) Sapajus spp., and 28 (1.6\%) Callicebus spp. Alouatta and Sapajus species had higher proportions of males than did Callithrix and Callicebus spp. $(\mathrm{p}<0.01)$. We compiled a population profile, including sex and age distribution by genus (Appendix Table 2), and a spatial distribution of positive NWPs, all from São Paulo state, by IHC, qRT-PCR, or both (Appendix Figure 1).

All cases had IHC results. Of 1,752 cases, 468 (26.7\%) were positive for YF: $437(61.7 \%)$ of $708 \mathrm{Al}$ ouatta spp., 8 (28.6\%) of 28 Callicebus spp., 13 (13.7\%) of 95 Sapajus spp., and 10 (1.1\%) of 921 Callithrix spp. Of the remaining cases, $1,171(66.8 \%)$ were negative and $113(6.4 \%)$ had inadequate results because of autolysis or absence of liver samples. No age $(p=0.55)$ or sex $(p=0.72)$ difference between positive and negative groups was detected.

Microscopic evaluation of IHC-positive cases identified 432 (92.3\%) of 468 cases that had a full spectrum of YF hepatic lesions (Figure 1). Three had other histologic patterns: $2(0.4 \%)$ had apoptotic hepatocytes, and $1(0.2 \%)$ had mild degenerative and reaction findings. These 3 cases were Alouatta spp. (Appendix Figure 2). Among all positive cases, 33 (7.1\%) had autolysis that impaired histologic classification; these cases were excluded from further analysis.

Of all cases evaluated, 1,193 (68.1\%) of 1,752 had qRT-PCR results, and 277 (23.2\%) of those had detectable YFV: 243 (64.5\%) of 374 Alouatta spp., 5 (31.2\%) of 16 Callicebus spp., 5 (7.5\%) of 67 Sapajus spp., and 24 (3.3\%) of 736 Callithrix spp. Some cases had inadequate IHC results because of autolysis/decomposition: 2 Callithrix spp. and 12 Alouatta spp. We found that $24(8.7 \%)$ of $277(8.7 \%)$ qRT-PCR-positive cases had negative results by IHC: 15/24 Callithrix spp. (62.5\%), 8/243 Alouatta spp. (3.3\%) and 1/5 Callicebus spp. (20\%). These cases were called discordant cases, and the remaining cases were called concordant cases.

The discordant group represented, excluding autolyzed tissues, $15(68.2 \%)$ of 22 positive Callithrix spp. by qRT-PCR, and $8(3.5 \%)$ of 231 positive Alouatta spp. This group included 15 cases with mild degenerative and reactional findings, 4 with steatosis, 1 with non-YF-associated hepatitis, 2 with apoptotic hepatocytes, 1 with multinucleation of hepatocytes, and 1 with no lesions. One case in a Sapajus spp. that had a positive IHC result and a negative qRT-PCR result was not considered for statistical analysis because of intense autolysis that impaired morphologic analysis.

Regarding viral load, Callithrix spp. had a higher Cq (median 34, range 9-37) than other genera $(p<0.001)$. There was no significant difference in $\mathrm{Cq}$ for Alouatta, Callicebus, and Sapajus spp. The discordant group had a higher Cq (median 34, range 12-37) than the concordant group (median 12, range 5-35) $(p<0.001)$. When we compared viral load in the concordant group, we observed no difference for NWP genera. We compared $\mathrm{Cq}$ values for the concordant and discordant groups (Figure 2) and the distribution of Cq values for Alouatta and Callithrix spp. and humans (Appendix Figures 3-5).

We calculated YF-associated proportional mortality rates on the basis of cases with liver antigen detected by IHC divided by all cases and YF proportional infections as number of cases with detectable YF virus by qRT-PCR divided by all cases. Callithrix sp. was the only genus with difference between proportional YF-related mortality rates and infection 


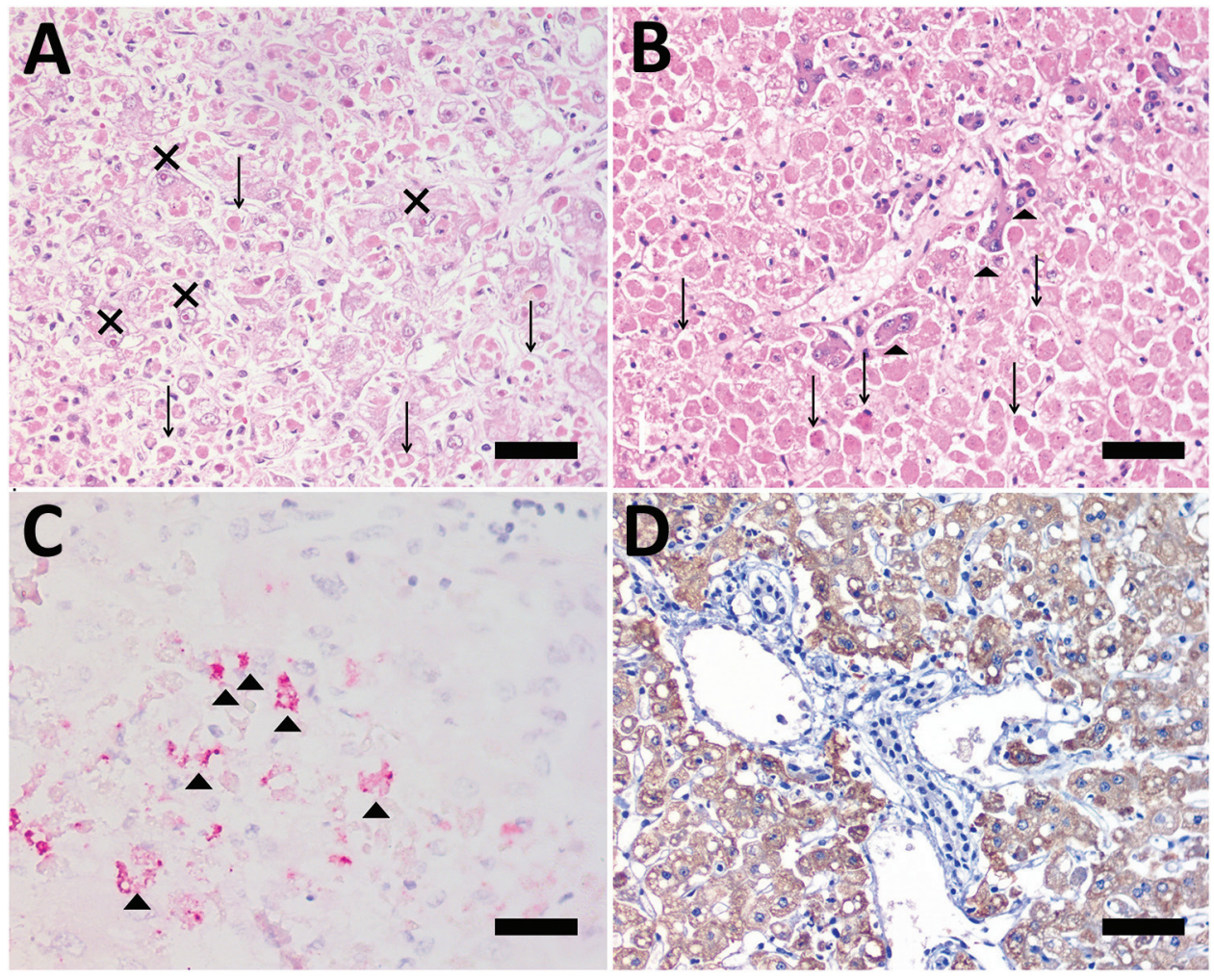

Figure 1. Photomicrographs of liver from human patient $(A, C)$ and Callicebus spp. monkey (B, D) with full spectrum of yellow fever (YF)-associated lesions, Brazil. Midzonal necrosis with multiple Councilman-Rocha Lima bodies (arrows), mild steatosis, and hepatocytes with eosinophilic nucleoli (Torres body [Xs]) (hematoxylin and eosin stained). B) Necrosis/apoptosis (diffuse and panlobular), associated with multiple Councilman-Rocha Lima bodies (arrows) and a few remaining viable hepatocytes (arrowheads) in periportal area (hematoxylin and eosin stained). C) Positive, multifocal immunolabeling for YF antigen (arrowheads) (antiYF, Warp red, counterstained with hematoxylin). D) Intense and diffuse immunolabeling for YF antigen (anti-YF, 3,3'-diaminobenzidine counterstaining with hematoxylin). Scale bars indicate $50 \mu \mathrm{m}$.

$(p=0.01)$, and proportional YF-related mortality rates and infection were different for NWP genera $(p<0.01)$ (Table 1).

\section{Humans}

We received 93 specimens from human case-patients who had suspected YF, 68 (73.1\%) men and $25(26.9 \%)$ women. Of these, $48(51.6 \%)$ had detectable YFV by qRT-PCR, $40(83.3 \%)$ men and $8(16.7 \%)$ women. Of these 48 patients, $46(95.8 \%)$ were IHC positive (concordant) and $2(4.7 \%)$ were IHC negative (discordant). The mean \pm SD age for positive case-patients by qRT-PCR ( $48.8 \pm 14.9$ years) was higher than for negative case-patients $(41.5 \pm 18.8$ years $)(p=0.04)$, and a higher prevalence was found in men $(p=0.03)$. The mean \pm SD time in days between onset of clinical signs and time of death (TOD) was $10.7 \pm 4.8$ (minimum 2 days, maximum 26 days). There was a direct correlation between TOD and Cq $(\rho=0.37 ; p=0.007)$, indicating an inverse correlation between TOD and viral load (Appendix Figure 6).

All IHC-positive cases showed a full spectrum of YF-associated lesions; 2 discordant cases had other histologic patterns (Appendix Table 3, Figure 7). Regarding viral load, humans had a median Cq of 20 (range 12-38). Human patients had significantly higher Cq values than NWPs in general $(p<0.001)$, except when compared with Callithrix spp. When we compared human concordant and NWP concordant cases, humans had a higher $\mathrm{Cq}$ than all genera of NWP. We compiled the distribution of $\mathrm{Cq}$ values for different NWP genera and humans (Figure 3 ) and the main characteristics of positive cases among humans and NWPs (Table 2).

\section{Comparison of Methods}

Histopathologic examination showed high sensitivity and specificity for most genera of NWPs and for human specimens if one considers the full spectrum of YF-associated lesions as positive results (Table 3). Agreement between qRT-PCR and IHC for detection of YFV was good for NWPs $(\kappa=0.936,95 \%$ CI $0.911-$ 0.961 ) and humans ( $\kappa=0.957,95 \%$ CI $0.898-1.000)$. Analysis of NWPs by genera showed that Callithrix spp. had lower agreement $(\kappa=0.475,95 \%$ CI $0.251-$ 0.698 ) than other genera.

The detection limit for IHC was calculated by using receiver operating characteristic curve analysis and considered the $\mathrm{Cq}$ value for liver samples. For NWPs in general, the cutoff value was 21 (sensitivity $99.2 \%$, specificity $95.6 \%$ ). For Alouatta spp., the cutoff value was 21 (sensitivity $99.1 \%$, specificity $87.5 \%$ ); for Callithrix spp., the cutoff value was 12 (specificity and sensitivity $100 \%)(p<0.0001)$. 


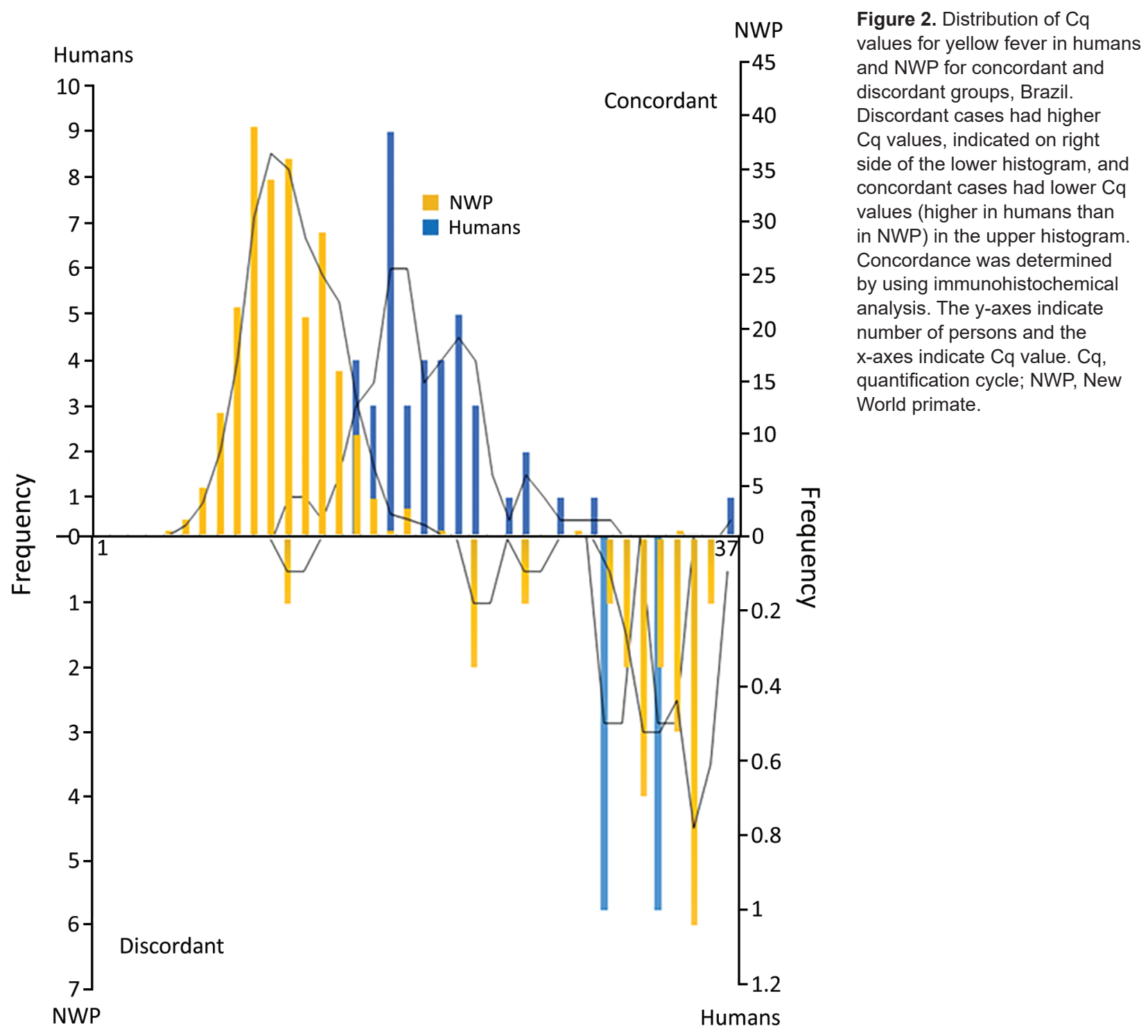

\section{Discussion}

A major epidemic of YF occurred in Brazil during 2016-2018. This epidemic had a dramatic progression in São Paulo state, and virus circulated in areas without vaccination coverage $(15,19)$. Wildlife surveillance is a pivotal tool for understanding arbovirus dynamics, despite difficulties in obtaining high-quality samples for analysis (20). A nonhuman primate (NHP) (rhesus monkey) is a useful model for YF (21) because it shows lesions similar to those in humans, although comparative studies among animals and humans, especially in an epidemic context, have not been conducted.

We analyzed a specific set of human samples that had a profile compatible with a sylvatic cycle: predominance of middle-age men, as proposed elsewhere (19). During 2012-2014, a YF outbreak occurred in the South Omo Zone in Ethiopia, which also had a higher mortality rate for adult men (22).

\begin{tabular}{|c|c|c|c|}
\hline Genus & Mortality rate, no. IHC positive/no. tested (\%) & $\begin{array}{c}\text { Infection rate, no. IHC or qRT-PCR } \\
\text { positive/no. tested (\%) }\end{array}$ & $p$ value \\
\hline Alouatta & $437 / 708(61.72)$ & $445 / 708(62.85)$ & 0.8 \\
\hline Callicebus & $8 / 28(28.57)$ & $9 / 28(32.14)$ & 1 \\
\hline Callithrix & $10 / 921(1.09)$ & $25 / 921(2.71)$ & 0.015 \\
\hline Sapajus & $13 / 95(13.68)$ & $13 / 95(13.68)$ & 1 \\
\hline
\end{tabular}

${ }^{*} \mathrm{IHC}$, immunohistochemical analysis; NWPs, New Word primates; qRT-PCR, quantitative reverse transcription PCR. 


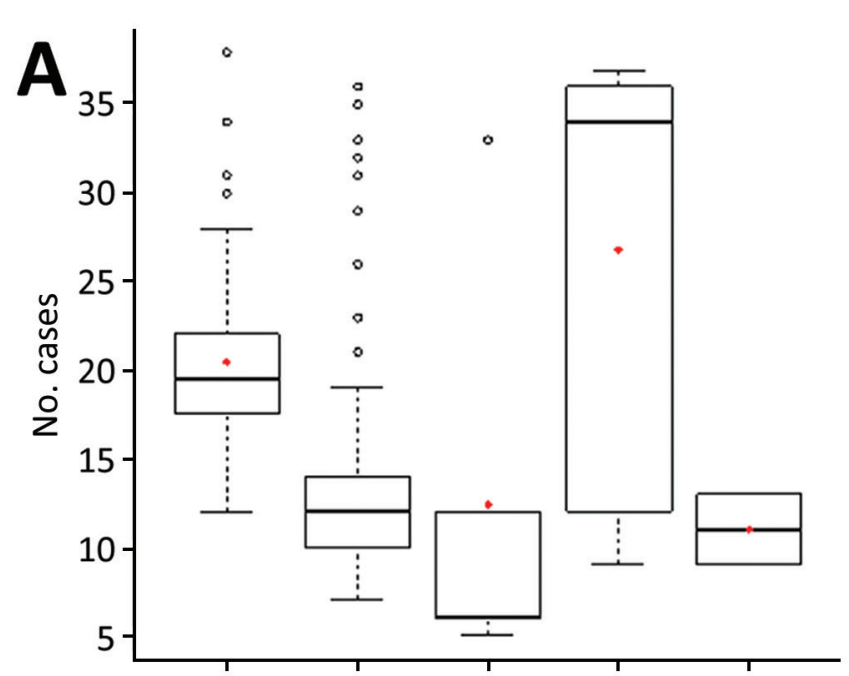

Humans Alouatta Callicebus Callithrix Sapajus

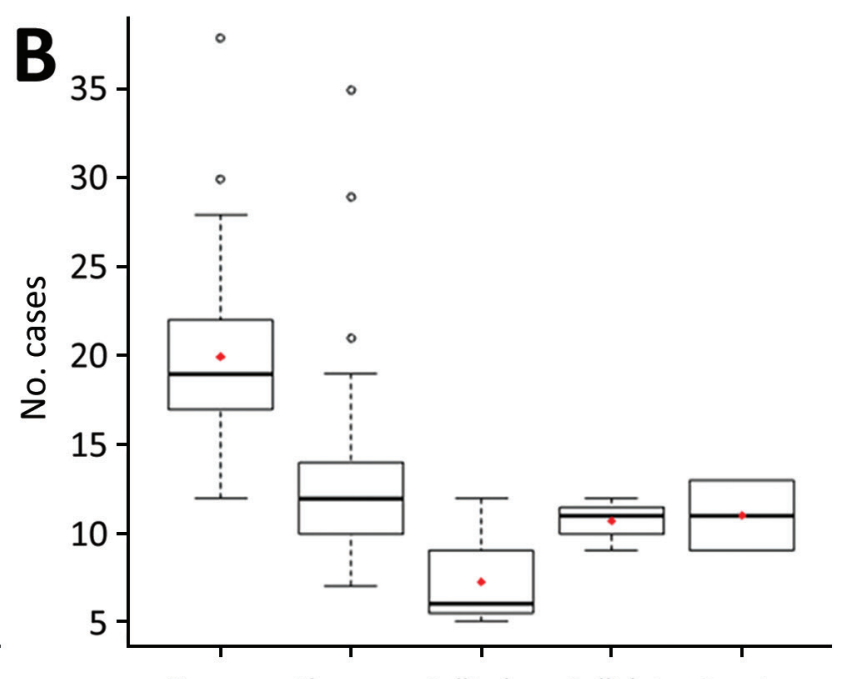

Humans Alouatta Callicebus Callithrix Sapajus

Figure 3. Distribution of $\mathrm{Cq}$ values for yellow fever for concordant and discordant cases $(A)$ and cases in different New World primates and in humans (B), Brazil. Box plots indicate Cq values among the groups, indicated by the $y$-axes, minimum, first quartile, third quartile, and maximum $\mathrm{Cq}$ values. Horizontal bars indicate medians. Circles indicate outlier Cq values, and red diamonds indicate mean $\mathrm{Cq}$ values. Concordance was determined by using immunohistochemical analysis (lesions and viral antigen in hepatocytes).

However, other urban arboviral disease outbreaks, such as dengue, show similar proportions in both sexes and a lower median age (23).

In NWPs, there was no difference in sex and age group distribution between positive and negative cases for YF, although adult males, especially from the genus Alouatta, were the most affected. Moreno et al. (24) observed the same profile in an outbreak among NWPs in Argentina, which highlighted the impact of conservation and reduction of population growth and mortality rates once adults have an effect on population reproductive capacity and provide care of young animals.

Most human samples had a traditional histologic pattern (positive results for IHC) consistent with YF $(1,13,14)$. Similarly to NWPs, we found discordant results for human case-patients who had lower viral loads, 1 of them limited by histologic over-fixation. Fixation periods $>1$ week might reduce immunoreactivity in tissues, even for robust antigens, such Ki-67 (25). In case-patients who had low viral loads and probably low amounts of antigen, these factors might have a higher negative effect. A direct correlation between TOD and Cq was observed for humans. This finding could explain the higher $\mathrm{Cq}$ values for humans than for NWPs because animals were mostly free living and were found dead without having any previous veterinary assistance. Also, the higher viral load among NWPs might indicate the higher potential of them as a group to act as amplifiers compared with humans.
Alouatta spp. accounted for $>90 \%$ of IHC-positive cases; this genus also had the highest proportional mortality rate attributable to YF and was the most affected animal group. Alouatta spp. are reported as being highly sensitive to YFV and the major sentinel in YF epidemics; epizootics have been reported in Venezuela (12) and Argentina (24), which affected howler monkeys and humans. In Brazil during the outbreak of 2008-2009, thousands of howler monkeys died in the southern region (26). There was also a major reduction in populations of these animals. In São Paulo state (8), both situations led to expansion of the YF vaccine coverage area.

Callicebus spp. is one of the most diverse genera of NWP and widely distributed in South America (27). Although these monkeys have been described as being resistant to YF (26), there are a few reports of YFV, including a genomic sequence extracted from a virus isolated from a monkey of this genus (28). We found no information regarding mortality rates or histologic pattern of lesions in the literature. In our study, Callicebus spp. monkeys were sensitive to YFV and developed a full spectrum of liver lesions, similar to those in humans, and a high viral load. These manifestations also occurred for Sapajus spp. monkeys.

Callithrix spp. overrepresented the analyzed animals, although only $1.1 \%$ of them died from YF. Callithrix is a genus widely distributed in São Paulo state and contains a vulnerable native species, $C$. aurita, a species introduced from northeastern Brazil; $C$. jacchus and C. penicillata, which were reeintroduced 
Table 2. Summary of main findings of human and NWP cases of yellow fever, Brazil*

\begin{tabular}{|c|c|c|c|c|c|}
\hline \multirow[b]{2}{*}{ Category } & \multirow[b]{2}{*}{ Human } & \multicolumn{4}{|c|}{ NWP genus } \\
\hline & & $\begin{array}{c}\text { Alouatta spp., } \\
n=445\end{array}$ & $\begin{array}{c}\text { Sapajus spp., } \\
n=13\end{array}$ & $\begin{array}{c}\text { Callithrix spp., } \\
n=25\end{array}$ & $\begin{array}{c}\text { Callicebus spp. } \\
n=9\end{array}$ \\
\hline \multicolumn{6}{|l|}{ Sex } \\
\hline M & 40 & 208 & 5 & 9 & 3 \\
\hline $\mathrm{F}$ & 8 & 79 & 5 & 5 & 5 \\
\hline \multicolumn{6}{|l|}{ Age } \\
\hline \multicolumn{6}{|l|}{ Humans, y } \\
\hline $0-19$ & 0 & & & & \\
\hline $20-30$ & 5 & & & & \\
\hline $31-45$ & 13 & & & & \\
\hline $46-65$ & 24 & & & & \\
\hline$>65$ & 7 & & & & \\
\hline \multicolumn{6}{|l|}{ NWP, category } \\
\hline Cub & & 18 & 0 & 1 & 0 \\
\hline Young & & 39 & 4 & 0 & 3 \\
\hline Adult & & 165 & 5 & 11 & 4 \\
\hline \multicolumn{6}{|l|}{ Hepatic histology† } \\
\hline Full spectrum of YF-associated lesions & 45 & 403 & 12 & 9 & 8 \\
\hline Incomplete spectrum of YF-associated lesions & 3 & 11 & 0 & 15 & 1 \\
\hline \multicolumn{6}{|l|}{ IHC and RT-qPCR profile } \\
\hline Concordant & 46 & 223 & 5 & 7 & 4 \\
\hline Discordant & 2 & 7 & 0 & 14 & 1 \\
\hline \multicolumn{6}{|l|}{ Quantification cycle } \\
\hline$<10$ & 0 & 73 & 2 & 3 & 3 \\
\hline $11-20$ & 28 & 147 & 3 & 4 & 1 \\
\hline $21-30$ & 17 & 5 & 0 & 0 & 0 \\
\hline$>30$ & 3 & 5 & 0 & 14 & 1 \\
\hline
\end{tabular}

${ }^{*} \mathrm{IHC}$, immunohistochemical analysis; NA, not applicable; NWPs, New Word primates; RT-qPCR, reverse transcription quantitative PCR. †Cases with marked autolysis were excluded.

from the savannah; and natural hybrids (29). Regarding YF, most experimental studies date from the 1930s and 1940s in which YFV was transmitted from inoculated C. penicillata monkeys to rhesus monkeys through mosquitos. Although some of the experimented animals had fevers and died, none of them had typical hepatic lesions of YF (30).

Knowledge about NWP hosts and their susceptibility to YFV could help clarify the potential of maintenance of virus in interepidemic periods among these different genera and anticipate new spillovers or establishment of enzootic cycles. It is useful to consider behavioral changes in human and NWP populations, such as anthropic invasion in natural areas, frequently in forest areas; intensification of agricultural activities; and presence of some genera of NWPs in urban areas. Santos et al. (31) reported that $C$. penicillata monkeys are capable of adapting to urban environments; can keep natural behavior and group sizes; and adapt to food sources normally found in these areas. In our study, we found positive callitrichids in urban or periurban areas, as well as Alouatta spp. monkeys in parks within urban areas, such as São Paulo. Therefore, the traditional division into 2 YF cycles (sylvatic and urban) might be insufficient or inaccurate to describe the complexity seen in this recent outbreak, and the coexistence of humans, NWPs, and vectors adapted to both groups in the same environment must be considered.

Valentine et al. discussed sylvatic cycles of arboviruses and highlighted the role of NHPs as hosts for chickungunya and Mayaro fever, although the possibility of other NHP reservoirs for both diseases is debated (32). The distinction among amplifiers and reservoirs in arbovirus cycles is complex, especially because of the acute nature of infection;

\begin{tabular}{|c|c|c|c|c|c|}
\hline Category & Sensitivity & Specificity & PPV & NPV & $\begin{array}{c}\mathrm{IHC} \text { and } \mathrm{qRT}-\mathrm{PCR} \\
\text { agreement, } \kappa\end{array}$ \\
\hline \multicolumn{6}{|l|}{ NWPs } \\
\hline All genera & $94.1(91.6-96.1)$ & $99.5(98.8-99.8)$ & $98.6(97-99.4)$ & $97.6(96.6-98.3)$ & $0.97(0.91-0.96)$ \\
\hline Alouatta & 97.3 (95.3-98.7) & 98.2 (95.6-99.5) & $99(97-99.4)^{\prime}$ & 95.3 (91.9-97.3) & $0.95(0.92-0.98)$ \\
\hline Callithrix & $37.5(18.8-59.4)$ & $99.9(99.3-100.0)$ & $90.0(54.3-98.6)$ & 98.2 (97.5-98.7) & $0.47(0.25-0.70)$ \\
\hline Sapajus & $100(73.5-100.0)$ & $100(94.4-100.0)$ & $100(94.4-100.0)$ & $100.0(94.4-100.0)$ & $0.90(0.70-1.00)$ \\
\hline Callicebus & $88.9(51.7-99.7)$ & 94.7 (74.0-99.9) & $88.9(53.9-98.2)$ & 94.7 (73.9-99.1) & $0.85(0.56-1.00)$ \\
\hline Humans & 97.9 (88.7-99.9) & $97.8(88.5-99.9)$ & $97.9(86.9-99.7)$ & $97.8(86.6-99.7)$ & $0.96(0.90-1.00)$ \\
\hline
\end{tabular}


short time for viremia and high mortality rates are characteristics not compatible with the classical reservoir definition (16).

Viral persistence in a host must not cause death or serious disease, and in short-term infections, such as YFV, the virus must find a new susceptible host within a host population to keep permanent circulation (33). Thus, arthropod vectors are considered the most suitable reservoir for arbovirus, and NHPs are commonly described as amplifiers (16). The profile exhibited by callitrichids in our study differed from those of other genera, and similar to our findings in dead animals, living animals might have low viremia with no clinical hepatitis. Consequently, the role of callitrichids as amplifiers must be questioned, and their potential as a reservoir population warrants further research.

São Paulo state has a strict range of callitrichid species: C. penicillata, C. jacchus, C. aurita, and hybrid Callithrix. These taxonomic variations might be related to differential patterns. A group of animals with high viral loads (similar to the other studied genera) and hepatic lesions could affect general susceptibility in a population. In a surveillance context, callitrichids have a minor role as sentinels because of their low prevalence, although they could be relevant in urban areas, if one considers how adapted they are to these environments and different they are from the other genera analyzed. Discordant cases represent a diagnostic and medical challenge that has clinical and epidemiologic implications, especially in NWPs, because there is usually no available clinical information, such as clinical signs and other laboratory findings (liver markers, blood count). We did not observe any specific liver lesion in these cases.

In our study, callitrichids represented most samples but showed the lowest positivity rates, which was consistent with results of laboratory methods. Better selection of animals for analysis based on geographic locations and host populations from other genera could guide economic and personnel resources and optimize surveillance.

The histologic pattern for YF, especially Councilman-Rocha Lima bodies, was described more than a century ago (34), and its role as a diagnostic tool has been reinforced in other reports $(13,14)$. In our study, histology was a sensitive tool for use with human and NWP samples; callitrichids were the exception. IHC for YF provided etiologic confirmation and high agreement with qRT-PCR results, even for autolyzed cases (3).

IHC and qRT-PCR had similar usefulness in diagnosing YF in an epidemic context. Furthermore, an inexpensive analysis, such as histopathologic analysis, could provide useful information, such as excluding negative cases, increasing laboratory response, and detecting other infectious diseases, some of them zoonoses. Moreover, histopathologic analysis, especially IHC, might be a useful screening examination because of its high sensitivity, low cost, and limited human resource requirements. The cases we report as discordant and positive cases with minimal histological lesions illustrate the usefulness of submission of complete epidemiologic and clinical information and samples from different organs for histologic analysis. In addition, a well-trained pathologist with experience in YF diagnosis in NWPs is needed to ensure a sensitive and specific diagnosis on the basis of histologic criteria.

Our study had some limitations. We could not identify species of NWPs, and differential susceptibility among members of the same genus is still unknown. We might have had bias related to sample representation because collection of carcasses was limited to areas near trails or roads. Also, this study was restricted to São Paulo state landscapes and its NWP diversity. Although the genera analyzed are distributed in other geographic areas, more studies with different neotropical primates are needed to ensure ideal surveillance.

In conclusion, NWPs are not a homogenous group and show differences regarding susceptibility, viral load, and proportional YF-associated mortality rates. Clarification of the differences within NWP genera and between NWPs and humans might help to improve and optimize strategic surveillance by directing laboratory resources to most susceptible genera and applying diagnostic tests in a more rational way.

\section{Acknowledgments}

We thank all professionals directly or indirectly involved in YF surveillance in São Paulo state; field and surveillance agents and laboratorial staff, especially those from the Centro de Vigilância Epidemiológica Prof. Alexandre Vranjac and the Pathology and Virology Center, Instituto Adolfo Lutz; Andreia Latorre for critically reviewing the manuscript, and Chris Gardiner for reviewing the English version of the manuscript.

The Yellow Fever Surveillance Program was supported by the Ministry of Health, Secretaria de Estado da Saúde, Coordenadoria de Controle de Doenças, the Adolfo Lutz Institute, and other public institutions. The postgraduate program was supported by Coordenação de Aperfeiçoamento de Pessoal de Nível Superior and the 
National Council for Scientific and Technological Development. J.D.-D. is a recipient of a postdoctoral fellowship from Fundação de Amparo à Pesquisa do Estado de São Paulo (grant 2017/02223-8).

N.C.C.A.F performed the literature search, evaluated IHC results and histopathology, analyzed data, and wrote the manuscript, J.M.G. evaluated IHC results and histopathology and reviewed the manuscript; J.D.-D. evaluated IHC results and histopathology; M.S.C. performed qRT-PCR and analyzed data; L.C.S. performed spatial analysis and tabulated results; S.D'A.I. evaluated IHC results and histopathology of human cases; R.A.R., C.S.C., and C.T.K. conducted IHC reactions; I.P.J. reviewed files and tabulated data, A.Y.M. and F.G.S.V. performed qRT-PCR; J.C. processed samples, reviewed files and tabulated data; L.J.T.A. analyzed IHC data; R.P.S. analyzed qRT-PCR data; J.S.N. analyzed human data; R.M.F.S. interpreted data; and J.L.C.D. mentored the work. All authors critically read the manuscript and agreed with the final version.

\section{About the Author}

Dr. Fernandes is a PhD student at the School of Veterinary Medicine and Animal Science, University of São Paulo, São Paulo, Brazil, and the lead veterinary pathologist responsible for yellow fever in NHPs at the Adolfo Lutz Institute, São Paulo, Brazil. Her primary

research interests are comparative pathology of human and NHP yellow fever-associated disease, and characterizing species-specific pathogenic mechanisms and factors modulating yellow fever-endemic cycles and triggering outbreaks among humans and NHPs in the Americas.

\section{References}

1. Monath TP, Vasconcelos PF. Yellow fever. J Clin Virol. 2015;64:160-73. https:/ / doi.org/10.1016/j.jcv.2014.08.030

2. Possas C, Lourenço-de-Oliveira R, Tauil PL, Pinheiro FP, Pissinatti A, Cunha RV, et al. Yellow fever outbreak in Brazil: the puzzle of rapid viral spread and challenges for immunisation. Mem Inst Oswaldo Cruz. 2018;113:e180278. https://doi.org/10.1590/0074-02760180278

3. Fernandes NC, Cunha MS, Guerra JM, Réssio RA, Cirqueira CD, Iglezias SD, et al. Outbreak of yellow fever among nonhuman primates, Espirito Santo, Brazil, 2017. Emerg Infect Dis. 2017;23:2038-41. https:/ / doi.org/10.3201/ eid2312.170685

4. Brazil Ministry of Health, Secretaria de Vigilância em Saúde, Departamento de Vigilância de Doenças Tranmissíveis. Guide to epizootics surveillance in non-human primates and entomology applied to the yellow fever surveillance [in Portuguese]. 2nd ed. Brasília: The Ministry; 2017.

5. Callender DM. Management and control of yellow fever virus: Brazilian outbreak January-April, 2018. Glob Public Health. 2019;14:445-55. https:// doi.org/10.1080/17441692.2018.1512144
6. Rylands AB, Mittermeier RA. Taxonomic listing of the new world primates. IUCN Species Survival Commission, Primate Specialist Group. Austin, TX; 2019 [cited 2020 Oct 7]. http://www.primate-sg.org/taxonomy

7. Catenacci LS, Ferreira M, Martins LC, De Vleeschouwer KM, Cassano CR, Oliveira LC, et al. Surveillance of arboviruses in primates and sloths in the Atlantic forest, Bahia, Brazil. EcoHealth. 2018;15:777-91. https://doi.org/10.1007/ s10393-018-1361-2

8. Moreno ES, Spinola R, Tengan CH, Brasil RA, Siciliano MM, Coimbra TL, et al. Yellow fever epizootics in non-human primates, São Paulo state, Brazil, 2008-2009. Rev Inst Med Trop São Paulo. 2013;55:45-50. https:/ / doi.org/10.1590/ S0036-46652013000100008

9. Rifakis PM, Benitez JA, De-la-Paz-Pineda J, RodriguezMorales AJ. Epizootics of yellow fever in Venezuela (20042005): an emerging zoonotic disease. Ann N Y Acad Sci. 2006;1081:57-60. https://doi.org/10.1196/annals.1373.005

10. Bensabath DG, Shope RE, Andrade AH, Souza AP. Recovery of Amarilico virus, coning from a single centinella, in the surroundings of Belem, Brazil [in Portuguese]. Bol. OPAS. 1966;60:187-92.

11. Leal SG, Romano AP, Monteiro RV, Melo CB, Vasconcelos PF, Castro MB. Frequency of histopathological changes in Howler monkeys (Alouatta sp.) naturally infected with yellow fever virus in Brazil. Rev Soc Bras Med Trop. 2016;49:29-33. https:// doi.org/10.1590/0037-8682-0363-2015

12. de Almeida MA, Dos Santos E, da Cruz Cardoso J, da Fonseca DF, Noll CA, Silveira VR, et al. Yellow fever outbreak affecting Alouatta populations in southern Brazil (Rio Grande do Sul State), 2008-2009. Am J Primatol. 2012;74:68-76. https:// doi.org/10.1002/ajp.21010

13. Vieira WT, Gayotto LC, de Lima CP, de Brito T. Histopathology of the human liver in yellow fever with special emphasis on the diagnostic role of the Councilman body. Histopathology. 1983;7:195-208. https://doi.org/ 10.1111/j.1365-2559.1983.tb02235.x

14. Quaresma JA, Barros VL, Pagliari C, Fernandes ER, Guedes F, Takakura CF, et al. Revisiting the liver in human yellow fever: virus-induced apoptosis in hepatocytes associated with TGF- $\beta$, TNF- $\alpha$ and NK cells activity. Virology. 2006;345:22-30. https://doi.org/10.1016/ j.virol.2005.09.058

15. Cunha MS, da Costa AC, de Azevedo Fernandes NC, Guerra JM, Dos Santos FC, Nogueira JS, et al. Epizootics due to yellow fever virus in São Paulo State, Brazil: viral dissemination to new areas (2016-2017). Sci Rep. 2019;9:5474. https:/ / doi.org/10.1038/s41598-019-41950-3

16. Kuno G, Mackenzie JS, Junglen S, Hubálek Z, Plyusnin A, Gubler DJ. Vertebrate reservoirs of arboviruses: myth, synonym of amplifier, or reality? Viruses. 2017;9:1-28. https:/ / doi.org/10.3390/v9070185

17. Alfaro JW, Silva JD Jr, Rylands AB. How different are robust and gracile capuchin monkeys? An argument for the use of Sapajus and Cebus. Am J Primatol. 2012;74:273-86. https://doi.org/10.1002/ajp.22007

18. Domingo C, Patel P, Yillah J, Weidmann M, Méndez JA, Nakouné ER, et al. Advanced yellow fever virus genome detection in point-of-care facilities and reference laboratories. J Clin Microbiol. 2012;50:4054-60. https:/ / doi.org/10.1128/ JCM.01799-12

19. Faria NR, Kraemer MU, Hill SC, Goes de Jesus J, Aguiar RS, Iani FC, et al. Genomic and epidemiological monitoring of yellow fever virus transmission potential. Science. 2018;361:894-9. https:// doi.org/10.1126/ science.aat7115 
20. Pandit PS, Doyle MM, Smart KM, Young CC, Drape GW, Johnson CK. Predicting wildlife reservoirs and global vulnerability to zoonotic Flaviviruses. Nat Commun. 2018;9:5425. https://doi.org/10.1038/s41467-018-07896-2

21. Engelmann F, Josset L, Girke T, Park B, Barron A, Dewane J, et al. Pathophysiologic and transcriptomic analyses of viscerotropic yellow fever in a rhesus macaque model. PLoS Negl Trop Dis. 2014;8:e3295. https:/ / doi.org/ 10.1371/journal.pntd.0003295

22. Mulchandani R, Massebo F, Bocho F, Jeffries CL, Walker T, Messenger LA. A community-level investigation following a yellow fever virus outbreak in South Omo Zone, South-West Ethiopia. PeerJ. 2019;7:e6466. https://doi.org/ $10.7717 /$ peerj. 6466

23. Miagostovich MP, Nogueira RM, Cavalcanti SM, Marzochi KB, Schatzmayr HG. Dengue epidemic in the state of Rio de Janeiro, Brazil: virological and epidemiological aspects. Rev Inst Med Trop São Paulo. 1993;35:149-54. https://doi.org/10.1590/S0036-46651993000200006

24. Moreno ES, Agostini I, Holzmann I, Di Bitetti MS, Oklander LI, Kowalewski MM, et al. Yellow fever impact on brown howler monkeys (Alouatta guariba clamitans) in Argentina: a metamodelling approach based on population viability analysis and epidemiological dynamics. Mem Inst Oswaldo Cruz. 2015;110:865-76. https://doi.org/Z10.1590/ 0074-02760150075

25. Sato M, Kojima M, Nagatsuma AK, Nakamura Y, Saito N, Ochiai A. Optimal fixation for total preanalytic phase evaluation in pathology laboratories: a comprehensive study including immunohistochemistry, DNA, and mRNA assays. Pathol Int. 2014;64:209-16. https://doi.org/10.1111/ pin.12164

26. Bicca-Marques JC, Freitas DS. Conservation letter: the role of monkeys, mosquitoes, and humans in the occurrence of a yellow fever outbreak in a fragmented landscape in south Brazil : protecting howler monkeys is a matter of public health. Access. 2010;3:78-89.
27. Hoyos M, Bloor P, Defler T, Vermeer J, Röhe F, Farias I. Phylogenetic relationships within the Callicebus cupreus species group (Pitheciidae: Primates): biogeographic and taxonomic implications. Mol Phylogenet Evol. 2016;102:20819. https:// doi.org/10.1016/j.ympev.2016.05.031

28. Rezende IM, Sacchetto L, Munhoz de Mello É, Alves PA, Iani FC, Adelino TÉ, et al. Persistence of yellow fever virus outside the Amazon Basin, causing epidemics in southeast Brazil, from 2016 to 2018. PLoS Negl TropDis. 2018;12:e0006538. https://doi.org/10.1371/journal.pntd.0006538

29. Nogueira DM, Ferreira AM, Goldschmidt B, Pissinatti A, Carelli JB, Verona CE. Cytogenetic study in natural hybrids of Callithrix (Callitrichidae: Primates) in the Atlantic forest of the state of Rio de Janeiro, Brazil. Iheringia Ser Zool. 2017;101:156-60. https:// doi.org/10.1590/ S0073-47212011000200002

30. Davis NC. The susceptibility of marmosets to yellow fever virus. J Exp Med. 1930;52:405-16. https://doi.org/10.1084/ jem.52.3.405

31. Santos M, Duarte M, Young R. Behavioural and ecological aspects of black tuftedear marmosets, Callithrix penicillata (Geoffroy, 1812) (Primates: Callitrichidae) in a semiurban environment. Revista de Etologis. 2014;13:37-46.

32. Valentine MJ, Murdock CC, Kelly PJ. Sylvatic cycles of arboviruses in non-human primates. Parasit Vectors. 2019;12:463. https:// doi.org/10.1186/s13071-019-3732-0

33. Mims CA. The meaning of persistent infections in nature. Bull World Health Organ. 1975;52:747-51.

34. Klion FM, Schaffner F. The ultrastructure of acidophilic "Councilman-like" bodies in the liver. Am J Pathol. 1966;48:755-67.

Address for correspondence: Natália C.C. de Azevedo Fernandes, Instituto Adolfo Lutz, Centro de Patologia, Av. Dr. Arnaldo, 355, CEP 01296000, Pacaembú, São Paulo, SP, Brazil; email: nccafernandes@yahoo.com.br 